\title{
Vehicle Swarm Rapid Prototyping Testbed
}

\author{
Emad Saad ${ }^{1}$, John Vian ${ }^{2}$, Greg J. Clark ${ }^{3}$, and Stefan Bieniawski ${ }^{4}$ \\ The Boeing Company, Seattle, WA, 98124
}

\begin{abstract}
Increased levels of vehicle collaboration and autonomy are seen as a means to reduce overall mission completion costs while expanding mission capabilities and increasing mission assurance for complex coupled system of systems. Systems health management technologies have made rapid advances that enable systems to know their own condition and capabilities, thus creating the opportunity for unprecedented levels of adaptive control, real-time reconfiguration, and mission contingency management. Multi-agent task allocation and mission managements systems must account for vehicle- and system-level health-related issues to ensure that these systems are reliable and cost effective to operate. Boeing's Vehicle Swarm Technology Lab (VSTL), established in 2004, includes a 100'x50'x20' testbed equipped with a vision-based motion capture indoor localization system. The testbed provides a cost-effective rapid prototyping capability for integrating health-based adaptive control of subsystems, vehicle, mission, and swarms to guarantee top-level system-of-systems performance metrics. The lab's heterogeneous fleet includes over 20 heterogeneous air vehicles, including VTOL and fixed wing, along with their ground stations and communication links in addition to heterogeneous ground vehicles and wall climbing robots. This paper discusses the Boeing VSTL design and capabilities, including the indoor localization system, multi-vehicle command and control (C2) and operator interface, realtime virtual environment, and health-based adaptive behaviors. The lab supports rapid prototyping and exploration of various multi-vehicle operational concept of operations and missions including persistent surveillance, area search and tracking, and high density air traffic management. Additionally, the lab supports experimentation tasks for many other platform configuration and collaborative air, ground, space, and maritime autonomous system of systems concepts.
\end{abstract}

\section{Nomenclature}

$\begin{array}{ll}3 D & =3 \text {-dimensional } \\ 6 D O F & =6 \text { degrees of freedom } \\ C 2 & =\text { command and control } \\ C O T S & =\text { commercial off the shelf } \\ h & =\text { height } \\ l & =\text { length } \\ N i M H & =\text { Nickel-Metal Hydride } \\ T C P & =\text { Transmission Control Protocol } \\ U A V & =\text { unmanned air vehicle } \\ U D P & =\text { Universal Datagram Protocol } \\ U G V & =\text { unmanned ground vehicle } \\ V S T L & =\text { Vehicle Swarm Technology Lab } \\ w & =\text { width } \\ S O C & =\text { state of charge }\end{array}$

\footnotetext{
${ }^{1}$ Engineer/Scientist, Boeing Research \& Technology, P.O. Box 3707 MC 42-53

${ }^{2}$ Technical Fellow, Boeing Research \& Technology, P.O. Box 3707 MC 42-53, AIAA Life Associate Fellow.

${ }^{3}$ Engineer/Scientist, Boeing Research \& Technology, P.O. Box 3707 MC 42-53.

${ }^{4}$ Engineer/Scientist, Boeing Research \& Technology, P.O. Box 3707 MC 42-51, AIAA Member.
} 


\section{Introduction}

$I_{c}^{s}$ NCREASED levels of vehicle collaboration and autonomy are seen as a means to reduce overall mission completion costs while expanding mission capabilities and increasing mission assurance ${ }^{1}$. Cooperative teams of autonomous vehicles exhibit many of the user-friendly, integrated, network-centric, intelligent, and adaptive characteristics desired for future system of systems concepts. Additionally, these systems stand to offer unprecedented levels of top-level functional performance when compared to today's generally single platform solutions. Additional technical advancements are needed to enable the high level of multi-vehicle collaboration and autonomy that are required to realize this vision. Impressive work is already proceeding in the areas of task planning, trajectory generation, de-confliction, sense and avoid, and robust control. Alternative approaches to health-based adaptive control are needed to guarantee achievement of a true top-level system of systems performance metric. Additionally, cost-effective means of rapid prototyping these system concepts need to be explored.

Systems health management technology has made rapid advances that enable systems to know their own condition and capabilities, thus creating the opportunity for real-time reconfiguration and mission adaptation. This self-awareness feature is seen as essential to achieving operational reliability as systems become more complex, highly-coupled, and autonomous. Additionally, accounting for human workload in a health-adaptive design approach will enable guaranteed safe UAV / UGV (Unmanned Air Vehicle / Unmanned Ground Vehicle) operations with varying levels of automation to accommodate the human operator. The resulting fine-grained characterization of real-time component and system performance limits will lead to a new era of control systems research that moves well beyond traditional adaptive methods.

While many researchers have been exploring autonomous multi-agent operations as discussed in Ref. 2 and Ref. 3, more work is needed on how to perform multi-agent health management for autonomous task groups. This includes the need for formal methods of health-based adaptive control across all levels (top-level discrete-event task

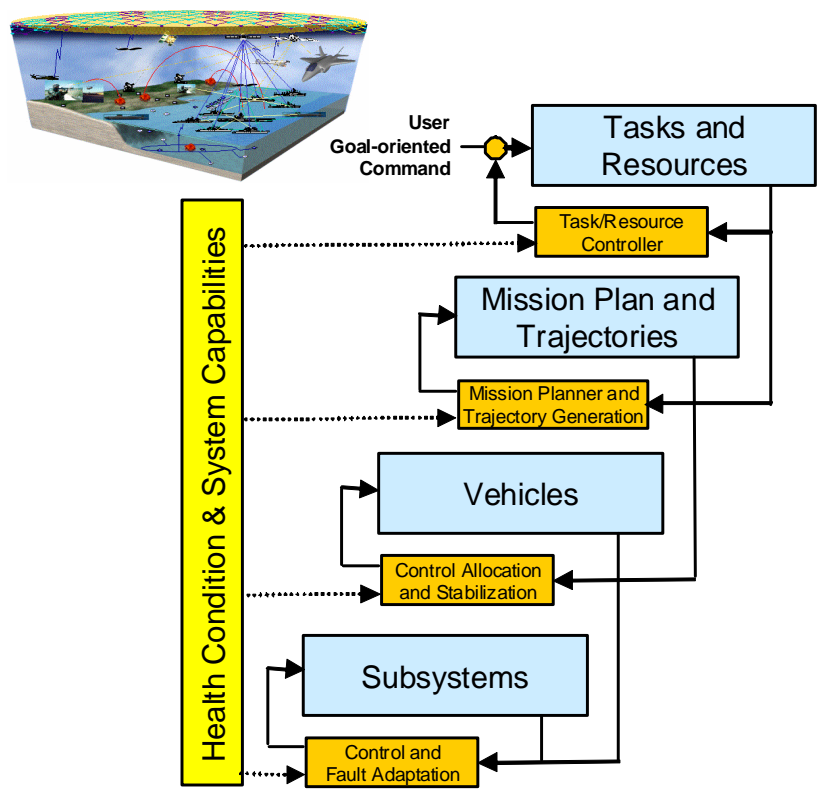

Figure 1. Health-based adaptive control architecture for hybrid system of systems. planning, platform-level mission system and guidance planning, control systems reconfiguration, and life-optimal subsystems adaptive control) to reliably perform complex tasks in real-time under realistic degraded conditions. Various architectures, as shown in Fig. 1, are being investigated to pursue this goal. Alternative approaches to credible rapid concept prototyping and algorithm testing are needed to develop autonomous collaborative systems in a cost-effective and timely manner.

In summary, multi-agent task allocation and mission managements systems must account for vehicle- and system-level health-related issues to ensure that these systems, together, are robust and cost-effective to operate.

\section{Rapid Prototyping}

As discussed in Ref. 4-6, many research groups have used a variety of platforms to verify advanced theories and approaches in the development of innovative UAV concepts as presented in Ref. 7-9. While pure simulation environments are very convenient, they typically do not enforce key real-world implementation challenges such as vehicle dynamics, determinism, communication delays, discretization effects, noise, processing latency, etc. Likewise, instrumenting radio-controlled aircraft and conducting authorized outdoor testing has proven to be costly and yields limited flight time for multi-vehicle mission algorithm development and assessment. Furthermore, these outdoor testbeds have several limitations that inhibit their utility for investigating the systems health management and adaptive control questions related to multi-day, multi-agent, mission operations. There is a clear need for an indoor system of systems testbed for rapidly reducing developmental risks, thus increasing technology readiness of vehicle swarms-related technologies, see Fig. 2. 
The Boeing Vehicle Swarm Technology Lab (VSTL) has recently been developed to provide lowcost alternatives for the rapid prototyping of mission algorithms, vehicle hardware, and health management ${ }^{10}$. The indoor testbed, shown in Fig. 2, is designed to use real hardware to examine autonomous system frameworks that include multi-vehicle systems health management, as well as issues related to centralized versus decentralized control. The testbed uses aerial and ground vehicles that operate autonomously in a large, indoor volume to execute various mission scenarios and evaluate different collaborative vehicle concepts of operation. Researchers can rapidly execute multiple mission scenarios in a short period of time with minimal setup and organization between tests.

With these capabilities, the VSTL testbed has been pivotal in the progress of Boeing's intelligent adaptive systems research. For example, in 2007, Boeing demonstrated the first known twelve vehicle autonomous flight with a single operator shown in Fig. 3. In addition, swarm control with a minimal number of operators has been enabled by implementing health-adaptive behaviors. This behavior includes vehicle and navigation state-based adaptation which increases flight safety. Safety bounds result in controlled actions, including normal landing, open-loop position graceful landing or thrust termination when things go wrong with a vehicle. A Boeing-developed swarm C2

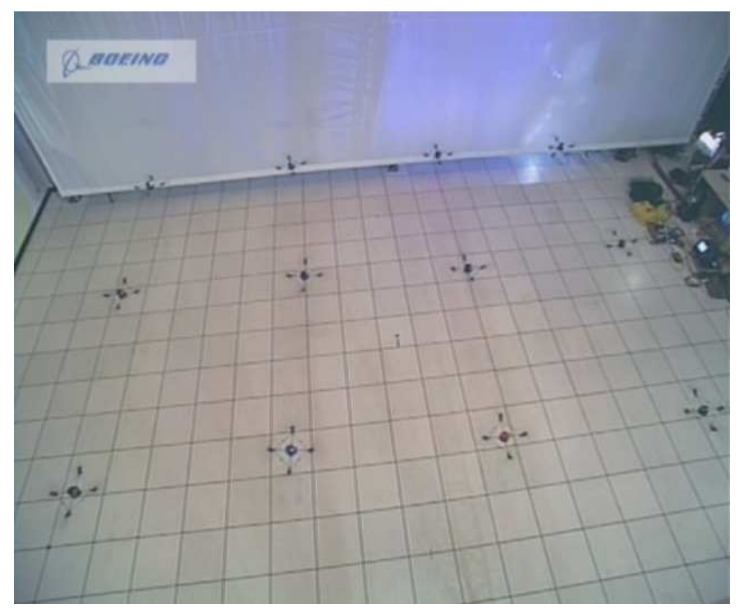

Figure 3. Twelve vehicle, single-operator, autonomous flight in the original 30 'x50'x30' VSTL facility.

approach, it has been possible to collaborate, investigate various advanced algorithms and swarms control methodologies, and quickly assess their potential value in an integrated system of systems setting.

The original 30 ' $\times 50$ ' $\times 30$ ' VSTL testbed was expanded in January 2009 to a larger 100'x50'x20' volume shown in Fig. 4 that will allow larger scale and more complex missions, additional numbers of autonomous vehicles, larger-sized vehicles, and expanded fixed wing flight operations. The lab is being equipped with access to Boeing's LabNet network service that provides connectivity to other Boeing labs worldwide, thus providing an opportunity for multi-site collaborative research, remote operations, and testing of

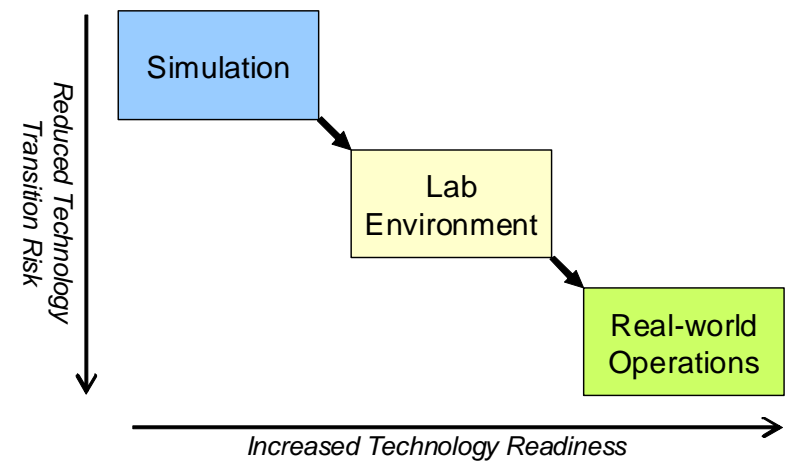

Figure 2. Indoor system of systems swarm technology lab environment testing is critical in the operational development risk-reduction path. (command and control) operator interface, called SwarmView, allows a single operator to simultaneously command multiple vehicles as well as monitor their state and health information. A real-time virtual environment vehicle dynamics.

Boeing started this rapid prototyping concept in 2004 through its Strategic Technology Area research initiative and has since worked with multiple universities to establish a streamlined research transition path. For example, Boeing initiated a collaboration with MIT in 2004 by providing an initial concept definition and funding, and later furnished a vision-based motion capture indoor localization system, Boeing common vehicle dynamic models, and hardware. Through this

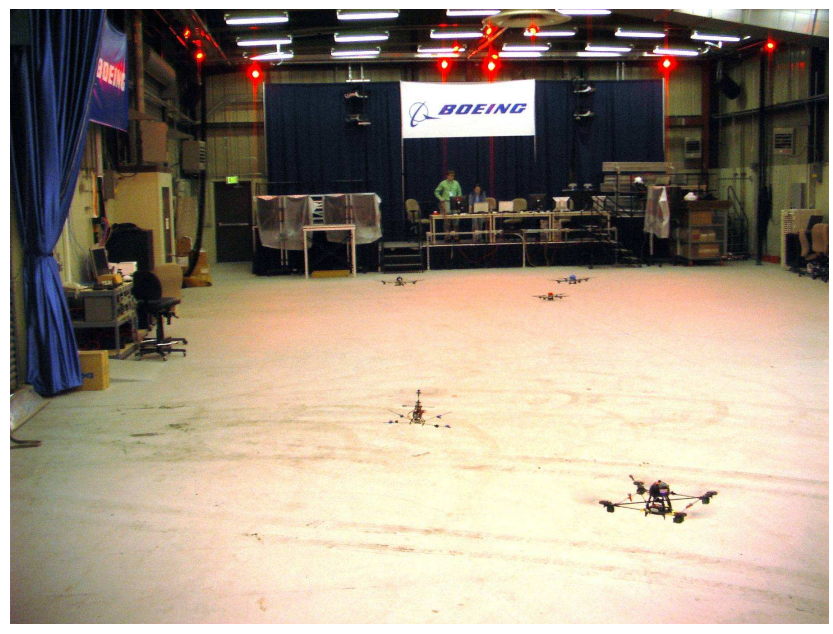

Figure 4. Boeing VSTL new 100'x50'x20' facility. provides $3 \mathrm{D}$ visualization of the swarm with $6 \mathrm{DOF}$ 
related issues such as latency.

The lab environment provides clean, low noise, low latency vehicle position, attitude, and communication data; however, the testbed provides a means of corrupting the vehicle or communications data to emulate real-world conditions to any desired degree. At the same time, certain characteristics and challenges associated with this rapid prototyping testbed may be exploited and studied for research purposes, such as the dependence of a vehicle's communication link for its own positional data in the lab versus the outdoor utilization of GPS navigation with onboard control.

The following sections provide an overview of the VSTL testbed architecture, the indoor localization system, the heterogeneous vehicle fleet, primary software components, and operator interfaces.

\section{VSTL Testbed Architecture}

The overall VSTL testbed architecture is comprised of hardware and software elements, as shown in Fig 5. Hardware elements include a high-accuracy, low-latency, vision-based, motion capture position reference system and a number of hardware vehicles under test. Each vehicle under test is equipped with its own on-board controller, health-monitoring, and communication sensor payload. While designed primarily for autonomous control, the architecture also permits real-time human operator in the loop control via a joystick or other pilot inceptor.

The software elements of the architecture include a vehicle position re-formatting and broadcasting application, a common ground-based vehicle control application for each of a number of vehicles under test, on-board vehicle control and communication applications. In addition, the architecture also supports concurrent real-time vehicle simulations interacting with actual real vehicle hardware while it is being operated within the testbed. Human operator interface software elements include a command and control application and a common situation awareness $3 \mathrm{D}$ virtual environment application. Communication between the various software elements is via either of two data buses. One bus is used for transmitting vehicle position and attitude data. The second bus is used for transmitting health, condition, and capability data as well as vehicle commands. The interaction between these applications is through UDP (User Datagram Protocol) Ethernet packets. The architecture also supports TCP (Transmission Control

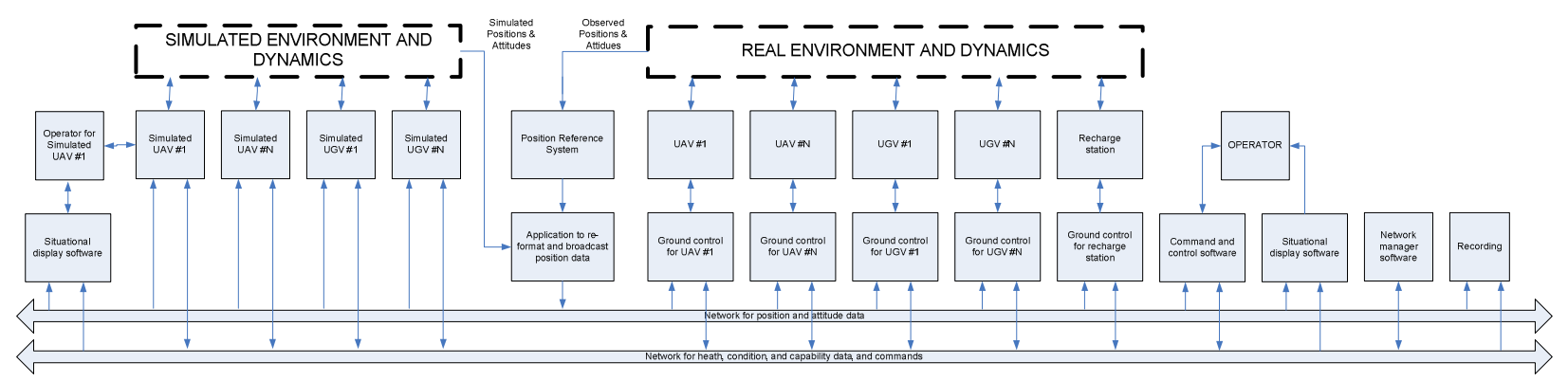

Figure 5. Boeing VSTL testbed hardware and software architecture.

Protocol) for less time-critical data when accurate data delivery is required.

\section{Localization System}

A commercial off the shelf (COTS) vision-based motion capture system from VICON is used to provide localization data in the form of position and attitude for all objects in the test volume. The system consists of a central data processing hub and a number of high-resolution digital cameras, each of which is equipped with local image processing, lens, and an LED strobe ring. The system generates coordinated pulses of visible light which are reflected from passive markers attached to objects of interest. The system then combines the returns seen by multiple cameras to triangulate the individual marker positions. Tracked objects are defined by equipping each object with a unique pattern of multiple markers which are then collectively recognized and tracked real-time by software. The localization system output is high-rate, high-accuracy measurements of position and attitude; position accuracy is sub-millimeter and angular accuracy is sub-degree. Data for multiple objects is provided at 100 frames per second with latency of approximately 10 milliseconds. 


\section{Heterogeneous Vehicles}

A collection of common, modular, vehicle hardware and software components have been developed as part of the VSTL testbed in order to expedite the autonomous vehicle integration process ${ }^{11}$. Currently-available healthenabled vehicles are all battery-powered and include quadrotor helicopters, coaxial blade helicopters, conventional helicopters, fixed-wing, ground crawling vehicles, and wall-climbing robots, refer to Fig. 6. One of two custom-built vehicle interface hardware packages can be applied to custom or commercially-available remote controlled vehicles, along rapid integration of new vehicles in the testbed. Both packages include a microprocessor loaded with common laboratory software, current sensors, voltage sensors, and a common laboratory communication system. The only difference between these packages lies in how the hardware interfaces with a given vehicle's actuators. That is, one package includes four motor speed controllers suitable for driving a vehicle's DC motors directly, whereas the other package includes four servo pulse outputs suitable for interfacing with the vehicle's commercially-available servos and speed controllers. These hardware packages fall within the UAV \#N block shown in Fig. 4. Additionally, vehicles may carry a variety of onboard sensor payloads, such

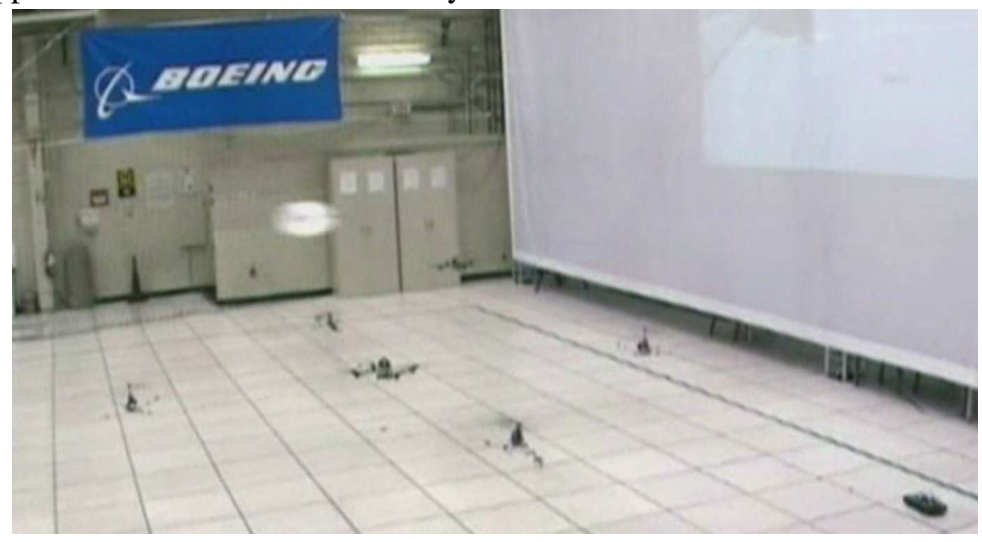

Figure 6. Heterogeneous vehicles including quadrotors, single blade helicopters, coaxial blade helicopters, a Disk fixed wing airplane, and a ground crawling vehicle performing a collaborative mission in the VSTL. as wireless on-board cameras which can be used for proof-of-concept demos such as search and track.

\section{A. Recharge Station}

The testbed also includes a recharge station for autonomous recharging of vehicle batteries, such as is required for persistent missions. The autonomous recharge station has been developed in collaboration with MIT and consists of a landing pad, a battery charger, and control circuitry to be able to remotely control battery recharge capability. The design of the landing pad guides the vehicles into place as it lands to ensure reliable electrical contact is made between the vehicle and the charger. Only minor modifications are required to make the helicopter or ground vehicles compatible with the recharge station. The control circuitry enables fully-autonomous remote

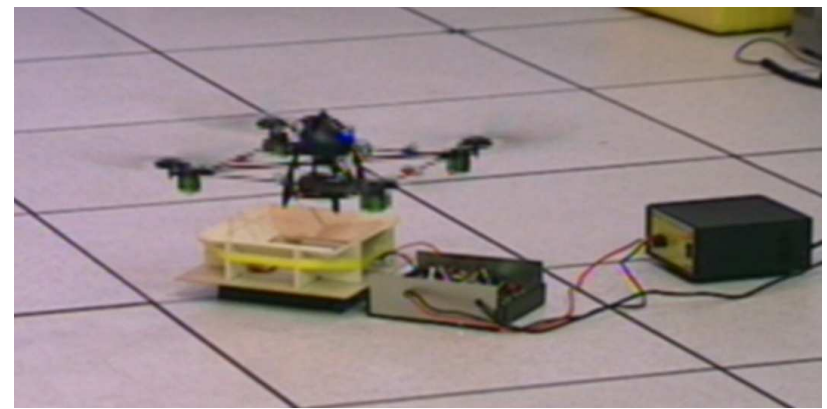

Figure 7. Autonomous Battery Recharge Station operation of the recharge station as illustrated in Fig. 7.

\section{Vehicle Health Monitoring}

The ability of a vehicle to autonomously monitor, assess, and act on the health of its various subsystems is essential for robust system and system of system operations. The vehicles used within the VSTL testbed are equipped with onboard sensing, computational, and communication capabilities which allow them to monitor and adapt to system degradations in real-time. Communication telemetry links enable the health information to be transmitted to a ground station for either bulk empirical data storage (such as for diagnostic / prognostic algorithm development and validation), real-time human operator command and control decision aiding, or to other vehicles for multi-vehicle, health-based, system adaptation and collaboration. Command and control station graphical userinterface functions enable real-time updates to fleet readiness status indicator charts as well as visualization of health data parameter trends. A standard common data format for health telemetry data files enables rapid processing using common engineering analysis tools such as MATLAB in addition to data archiving for diagnostic and prognostic 

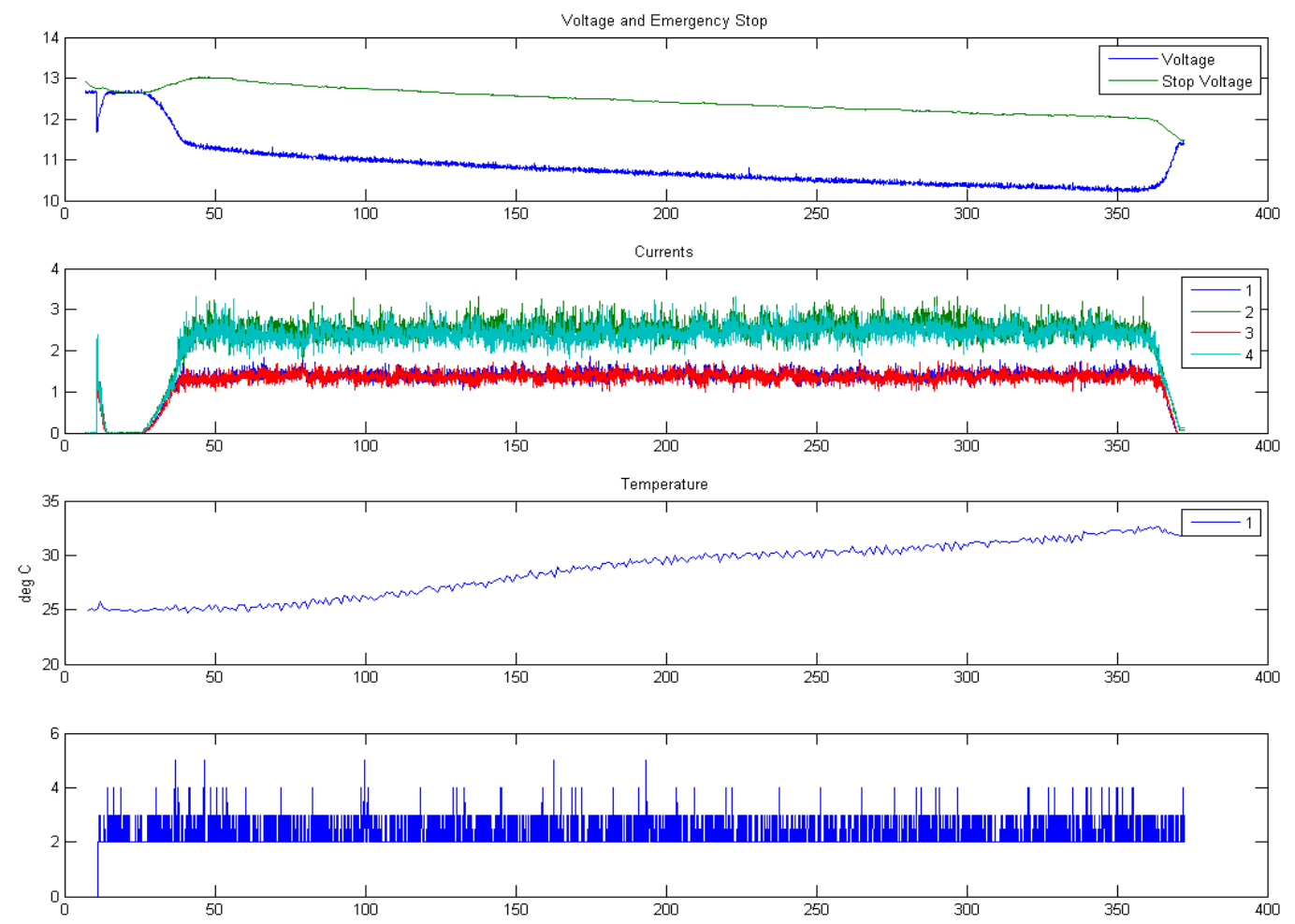

Figure 8. Real-time vehicle health data monitoring including battery voltage, motor currents, motor temperature, and communication link health.

algorithm development. Vehicle health data include battery voltage and SOC, motor currents, motor temperature, and communication link health.

\section{Adaptive Control and Supervision}

The VSTL testbed is architected using a health-adaptive design approach that enables guaranteed safe UAV/UGV operations, a fundamental requirement for testbed operations. This health-adaptive behavior includes adaptation to the communication health. For example, when communication is lost, the vehicle no longer receives information about its position and will become unstable. By monitoring real-time command communications latency, the vehicle can initiate a gyro-augmented (on-board) controlled landing as a result of loss of communication.

The vehicle flight safety system includes testbed-centric and vehicle-centric systems. Safe flight is ensured through navigation state-based adaptation, i.e. monitoring and adaptation based upon the guidance and control states. Layered protections against excessive position and attitude deviations have also been put in place. The first layer provides an autonomous limiting response, while the second provides an emergency response. For the position envelope the first limit consists of restricting the commanded position to a pre-specified volume. The second layer involves an immediate shutdown when the volume is further exceeded by a defined amount. Additionally, the vehicle's attitude deviation (from horizontal) is continually monitored as an indication of nominal flight behavior. When the attitude exceeds a specified threshold, indicative of a vehicle fault or excessive disturbances, the vehicle performs a controlled landing. A second layer results in an immediate shutdown if the angular excursion is even greater.

Vehicle state-based adaption also provides multiple levels of safety algorithms to protect the vehicle battery. Battery monitoring is especially important for high energy density rechargeable Lithium Polymer batteries which are more volatile than other rechargeable batteries such as NiMH. On-board software monitors current draw and loaded voltage to estimate the unloaded voltage of the battery. This is then used by an off-board algorithm to estimate the remaining flight time. This may then be used by an automated vehicle tasking process and is also displayed to the operator so that an appropriate manual action can be taken. The onboard algorithm uses a very low threshold on the unloaded voltage to shut off the vehicle as a last-minute fail-safe. 
Subsystem catastrophic event adaptation allows the vehicle to perform a graceful landing, instead of a simple thrust termination, in case of catastrophic events. While healthy, the vehicle ground software uplinks a collective command ramp-down profile designed to limit the ground impact velocity. Furthermore, the on-board controller continuously monitors the motor trim commands specific to the particular vehicle. When activated, graceful landing control applies the last known motor trim and collective ramp-down profiles. Additionally, on-board gyro feedback remains active to stabilize the vehicle's attitude during the decent. As a result, the vehicle strikes the ground with a specific impact velocity independent of the starting altitude.

\section{Health-adaptive Collision Avoidance and Real-time Deconfliction}

Collision avoidance is also essential for safe operation of multiple vehicles operating within the same space. Boeing developed a collision avoidance algorithm ${ }^{12}$ in collaboration with the University of Illinois at Urbana-Champaign. This algorithm currently provides guaranteed collision avoidance using mathematical proofs involving the vehicle and algorithm dynamics. This healthbased collision avoidance algorithm allows for adaptation of the avoidance parameters to changes in vehicle dynamics. The collision avoidance algorithm is designed to function both in autonomous, waypoint-commanded flight, and in operatorcommanded flight.

To prevent vehicles from approaching one another too closely the planned flight path of each vehicle can be deconflicted from one another. This involves looking along the planned path for possible conflicts and altering the path appropriately. This is then balanced by the desire to return to the originally planned path or waypoint. The current algorithm, developed by MIT and modified by Boeing, balances these two requirements to form an algorithm that has provided smooth, minimal deviations to result in robust deconfliction.

\section{VSTL Operator Interfaces}

The following sections describe VSTL operator hardware and software user interface capabilities.

\section{B. C2 Health Swarm Operator Interface}

SwarmView is a multi-vehicle command and control (C2) operator interface software application. It allows a single human operator to command and monitor multiple autonomous vehicles from a single workstation. The interface supports high-level mission commands, including waypoint and task commanding, as well as lowlevel operational commands such as controlling and verifying vehicle subsystem status. It also allows graphical and tabular monitoring of vehicle health and state information.

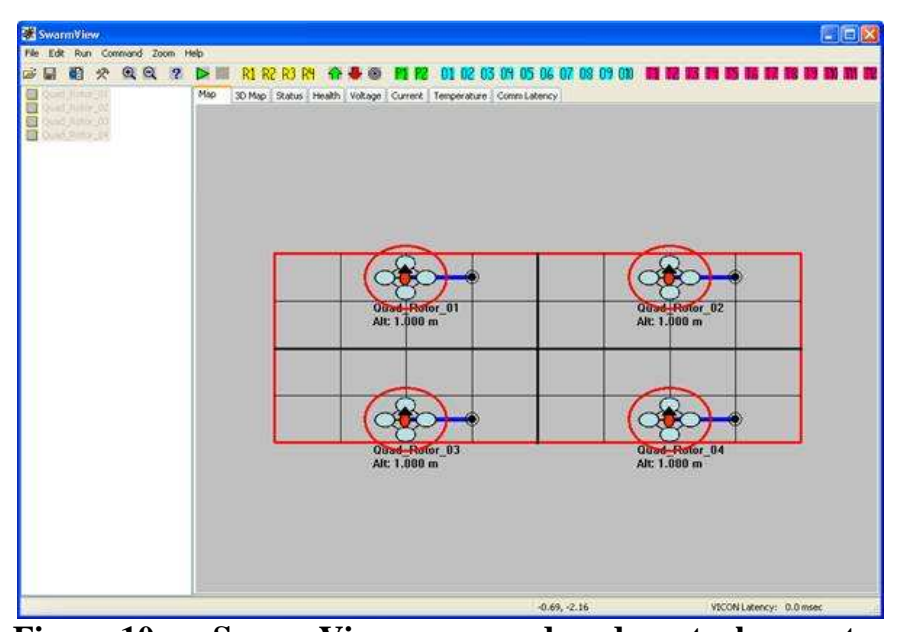

Figure 10. SwarmView command and control operator interface.

\section{Real-Time Virtual Environment}

A real-time virtual environment was built based on a software game engine to provide a 3D high-fidelity situational display for multiple heterogeneous vehicles. The display provides a customizable virtual environment 
(e.g. urban, indoor, forest, etc.) and includes vehicle data visualization (e.g. health and condition), as well as commanding the vehicle. The interface supports either simulation or real vehicles. This 3D interface includes different flight views including first person, third person, and top down. It provides several mission visualization aids including a Fog of War display for search missions, and flight traces. It allows viewing of $6 \mathrm{DOF}$ vehicle dynamics.

\section{Joystick Controller}

Using the SwarmView command interface, an operator can be given joystick control over any vehicle for a pre-determined amount of time. The joystick can be used to control the vehicle's $\mathrm{x}$ and

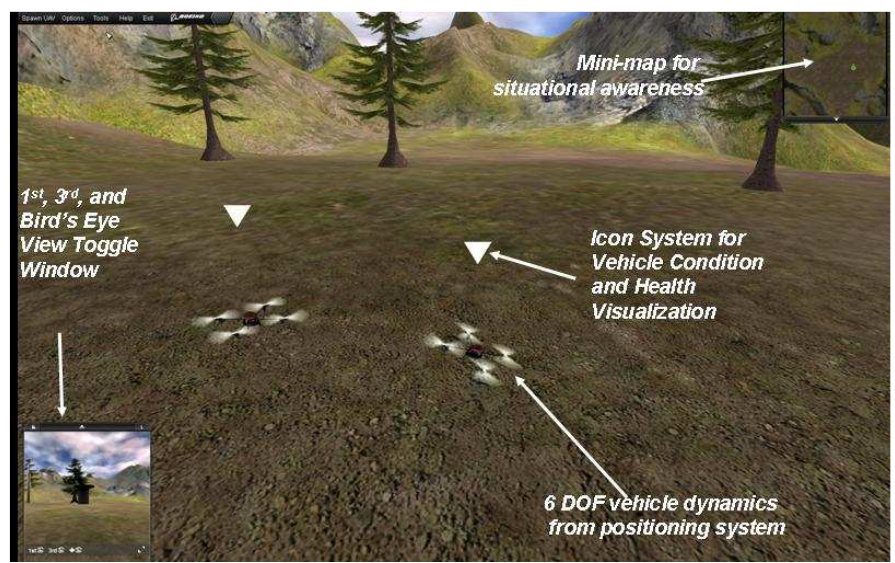

Figure 11. 3D virtual environment for real-time swarm visualization and concept of operations evaluation. $\mathrm{y}$ velocities, as well as its height and yaw position. If joystick input is not received, the vehicle will continue to hold its position. When the time limit has been reached, control is relinquished back to the autonomous vehicle controller. Force feedback on the joystick can also be used to alert operator of obstacles, via the collision avoidance algorithm.

\section{Applications}

To date, the VSTL testbed has been used to test and demonstrate several multi-vehicle health-adaptive missions ${ }^{13}$. Missions include persistent surveillance, area search, and high density air traffic management. In these example missions, we achieved higher level of autonomy and persistence through task and mission adaptation to the vehicle health and capabilities.

The persistent surveillance mission used a health-adaptive task planner in order to maintain persistence beyond the duration of an individual vehicle's battery life. The health-enabled vehicle hardware monitors the battery level and the health-adaptive task planner commands a vehicle to land whenever its battery level is below a set threshold. The task planner also keeps track of the other vehicles' battery conditions. It then automatically launches a vehicle with enough energy to replace the landing one in order to maintain the number of vehicles required for the surveillance coverage. Fig. 12 shows four vehicles performing persistent surveillance around an orbit with two vehicles on standby.

The area search mission uses a health-adaptive mission planner that re-plans whenever vehicle condition or capabilities change. When a vehicle is low on battery or is disabled for any reason, the mission planner re-plans the area search mission by reallocating the remaining search area among the remaining good vehicles in order to complete the area coverage and guarantee the required probability of detection. Figure 13 shows a heterogeneous fleet of five air and two ground vehicles performing a collaborative area search mission. Despite one of the air vehicles having an unplanned failure and not taking off, the mission planner dynamically re-planned by reallocating

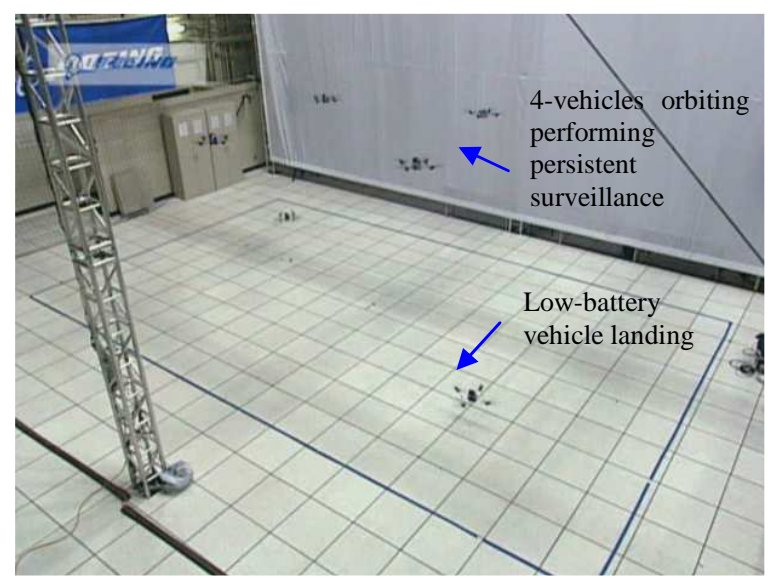

Figure 12. Multi-vehicle VSTL surveillance mission.

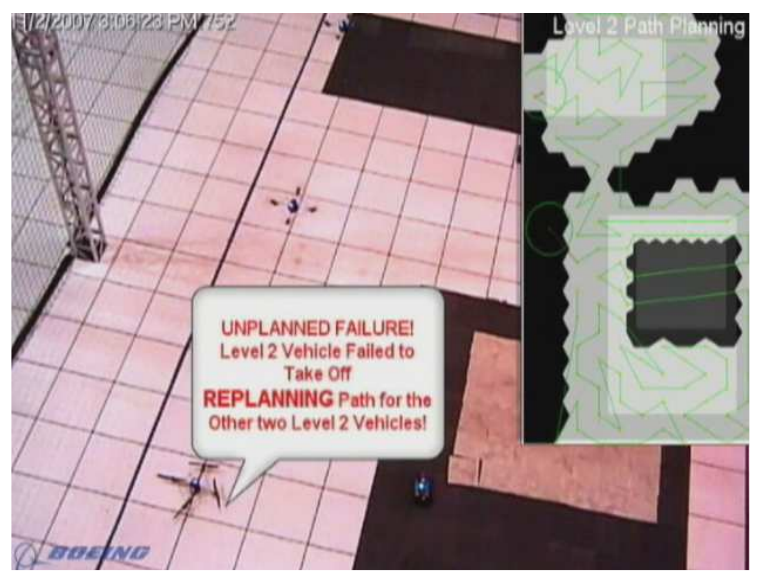

persistent Figure 13. the VSTL.

Health-adaptive area search mission in

8

American Institute of Aeronautics and Astronautics 
the aerial search area among the remaining air vehicles. This particular mission also included areas with different occlusion levels: clear, clouds, and foliage. The adaptive mission planner therefore allocated the search task to the different vehicles based on their sensor payload capability. For example, the ground vehicles covered the foliage area due to the foliage obstruction for the air vehicle sensors.

\section{Conclusion}

An indoor, multi-vehicle, flight testbed, such as the Boeing VSTL, provides a cost effective means for rapid prototyping of multi-vehicle mission planning, control, vehicle hardware and health-adaptive systems. This intermediate step between simulation and real-world operational testing provides further risk reduction of new technology development compared with only simulation validation. Health-based adaptation increases the level of autonomy in system of system operations under system and environmental uncertainty in order to achieve required mission assurance. Health-based adaptation extends to include adaptation to vehicle conditions and capabilities. Boeing is continuing development of the VSTL testbed to support experimentation and assessment of autonomous and collaborative system concepts for a wide variety of applications.

\section{References}

${ }^{1}$ Vian, J., and How, J., "Enabling Technology for Collaborative Autonomous Multi-vehicle Systems Development", White Paper Submitted in response to 2006 IEEE Conference on Decision and Control AFOSR/AFRL call for inputs on Guidance and Control for Military Systems: Current Practice and Future Directions.

${ }^{2}$ Gaudiano, P., Shargel, B., Bonabeau, E., and Clough, B., "Control of UAV SWARMS: What the Bugs Can Teach Us," Proceedings of the 2nd AIAA Unmanned Unlimited Systems, Technologies, and Operations Aerospace Conference, San Diego CA, September 2003.

${ }^{3}$ Paruanak, H., Brueckner, S., and Odell, J., "Swarming Coordination of Multiple UAV's for Collaborative Sensing," Proceedings of the 2nd AIAA Unmanned Unlimited Systems, Technologies, and Operations Aerospace Conference, San Diego CA, September 2003.

${ }^{4}$ Valenti, M., Bethke, B., Fiore, G,. How, J., and Feron, E., "Indoor Multi-Vehicle Flight Testbed for Fault Detection, Isolation, and Recovery," Proceedings of the AIAA Guidance, Navigation, and Control Conference and Exhibit, Keystone CO, August 2006.

${ }^{5}$ Valenti, M., Bethke, B., Dale, D., How, J., and Vian, J., "The MIT Indoor Multi-Vehicle Flight Testbed," Proceedings of IEEE International Conference on Robotics and Automation, Roma Italy, April 2007.

${ }^{6}$ Shim, D., Chung, H., Kim, H. J., and Sastry, S., "Autonomous Exploration in Unknown Urban Environments for Unmanned Aerial Vehicles," Proceedings of the AIAA Guidance, Navigation, and Control Conference and Exhibit, San Francisco CA, August 2005.

${ }^{7}$ King, E., Alighanbari, M., Kuwata, Y., and How, J. P., "Coordination and Control Experiments on a Multi-Vehicle Testbed," Proceedings of the IEEE American Control Conference, 2004.

${ }^{8}$ Nelson, D. R., Barber, D. B., McLain, T. W., and Beard, R. W., "Vector Field Path Following for Small Unmanned Air Vehicles," Proceedings of the 2006 American Control Conference, Minneapolis MN, June 2006.

${ }^{9}$ Hoffmann, G., Rajnarayan, D. G., Waslander, S. L., Dostal, D., Jang, J. S., and Tomlin, C., "The Stanford Testbed of Autonomous Rotorcraft for Multi Agent Control (STARMAC)," Proceedings of the 23rd Digital Avionics Systems Conference, Salt Lake City UT, November 2004.

${ }^{10}$ Vian, J. L., Provine, R. C., Bieniawski, S. R., Saad, E. W., Pigg, P. E. R., Clark, G. J., Mansouri, A. R., Abdel-Motagaly, K., Erignac, C. A., Troy, J. J., Murray, Paul, How, J. P., Valenti, M. J., Bethke, B. M., The Boeing Company, Chicago, IL, U.S. Patent Application for an "Autonomous Vehicle Rapid Development Testbed Systems and Methods," Pub. App. No. 20080033684, filed July 24, 2006.

${ }^{11}$ Halaas, D. J., Bieniawski, S. R., and Vian, J., Control and Management of an Indoor, Health Enabled, Heterogenous Fleet, Proceedings of the AIAA Infotech@Aerospace Conference and Exhibit and AIAA Unmanned...Unlimited Conference and Exhibit, AIAA-2009-2036, Seattle, WA, 2009.

${ }^{12}$ Valicka, C. G., Bieniawski, S. R., Vian, J., Stipanović, D. M., "Cooperative Avoidance Control for UAVs," Proceedings of IEEE $10^{\text {th }}$ International Conference on Control, Automation, Robotics and Vision ICARCV 2008, Hanoi, Vietnam, December 2008.

${ }^{13}$ Bieniawski, S. R., Pigg, P. E. R., Bethke, B., and Vian, J., "Exploring Health-Enabled Mission Concepts in the Vehicle Swarm Technology Laboratory," Proceedings of the AIAA Infotech@Aerospace Conference and Exhibit and AIAA Unmanned...Unlimited Conference and Exhibit, AIAA-2009-1918, Seattle, WA, 2009.

${ }^{14}$ Bieniawski, S., Halaas, D., and Vian, J., "Micro-Aerial Vehicle Flight in Turbulent Environments: Use of an Indoor Flight Facility for Rapid Design and Evaluation," Proceedings of the AIAA Guidance, Navigation and Control Conference and Exhibit 18 - 21 August 2008, Honolulu, Hawaii. 